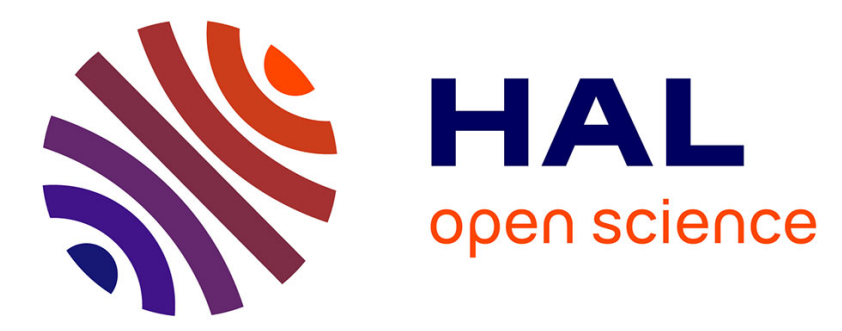

\title{
Stereo Autocalibration from One Plane
}

David Demirdjian, Andrew Zisserman, Radu Horaud

\section{To cite this version:}

David Demirdjian, Andrew Zisserman, Radu Horaud. Stereo Autocalibration from One Plane. 6th European Conference on Computer Vision (ECCV '00), Jun 2000, Dublin, Ireland. pp.625-639, 10.1007/3-540-45053-X_40. inria-00590132

\section{HAL Id: inria-00590132 \\ https://hal.inria.fr/inria-00590132}

Submitted on 3 May 2011

HAL is a multi-disciplinary open access archive for the deposit and dissemination of scientific research documents, whether they are published or not. The documents may come from teaching and research institutions in France or abroad, or from public or private research centers.
L'archive ouverte pluridisciplinaire HAL, est destinée au dépôt et à la diffusion de documents scientifiques de niveau recherche, publiés ou non, émanant des établissements d'enseignement et de recherche français ou étrangers, des laboratoires publics ou privés. 


\title{
Stereo Autocalibration From One Plane
}

\author{
David Demirdjian ${ }^{1}$, Andrew Zisserman $^{2}$, and Radu Horaud ${ }^{1}$ \\ 1 INRIA Rhône-Alpes, 655 av. de l'Europe, 38330 Montbonnot, France \\ david.demirdjian@inrialpes.fr, radu .horaud@inrialpes.fr \\ 2 Dept. of Engineering Science, University of Oxford, Oxford OX13PJ, England \\ az@robots.ox.ac.uk
}

\begin{abstract}
This paper describes a method for autocalibrating a stereo rig. A planar object performing general and unknown motions is observed by the stereo rig and, based on point correspondences only, the autocalibration of the stereo rig is computed. A stratified approach is used and the autocalibration is computed by estimating first the epipolar geometry of the rig, then the plane at infinity $\Pi_{\infty}$ (affine calibration) and finally the absolute conic $\Omega_{\infty}$ (Euclidean calibration). We show that the affine and Euclidean calibrations involve quadratic constraints and we describe an algorithm to solve them based on a conic intersection technique. Experiments with both synthetic and real data are used to evaluate the performance of the method.
\end{abstract}

\section{Introduction}

Autocalibration consists of retrieving the metric information of the cameras their internal parameters and relative position and orientation - from images, without using special calibration objects. Additional constraints can also be introduced such as knowledge of some of the internal parameters of the two cameras (aspect ratio, image skew, ...).

Planar autocalibration has several advantages. Planar scenes are very easy to process, enable very reliable point matching by fitting inter-image homographies, and very accurate estimation of the homographies. It will be seen that only the homographies are required for the autocalibration.

Many approaches for autocalibration have been developed for monocular and binocular sensors in recent years. Faugeras, Luong and Maybank [5] proposed solving the Kruppa equations from point correspondences in 3 images. However, this requires non-linear solution methods. An alternative is to first recover affine structure and then solve for the camera calibration from this. Such a "stratified" approach [4] can be applied to a single camera motion $[1,7,9,12,14]$ or to a stereo rig in motion $[2,10,20]$ and requires no knowledge of the observed scene. The stratified approach applied to the autocalibration of a stereo rig involves the computation of projective transformations of 3-D space, that is the projective 
transformation that maps two different projective reconstructions of the same 3 -D rigid scene. Unfortunately, these projective motions cannot be estimated when the 3-D scene is planar so those autocalibration approaches cannot be used.

Some approaches for calibration $[11,16,18]$ and autocalibration [17] from planar scenes have also been developed. In [17], the author uses the constraint that the projections of the circular points of a 3-D plane must lie on the image of the absolute conic. The proposed criteria is non-linear and the associated optimization process must be bootstrapped. Unfortunately no general method is given to obtain this bootstrapping.

We show here that, using a stereo rig, the stratified paradigm is very well adapted for autocalibration from planar scenes and extend the idea developed in $[17]$. We prove the following results:

(1) Affine calibration can be uniquely estimated from 3 views of a plane.

(2) Euclidean calibration can be uniquely estimated from 3 views of a plane if at least one of the cameras of the rig has zero image skew and known aspect ratio. Otherwise 4 views are required.

\section{Preliminaries}

\section{$2.1 \quad$ Camera model}

A pinhole camera projects a point $\boldsymbol{M}$ from the 3 -D projective space onto a point $\boldsymbol{m}$ of the 2-D projective plane. This projection can be written as a $3 \times 4$ homogeneous matrix $\mathbf{P}$ of rank equal to 3 :

$$
\boldsymbol{m} \simeq \mathbf{P} M
$$

where $\simeq$ is the equality up to a scale factor. If we restrict the 3 -D projective space to the Euclidean space, then it is well known that $\mathbf{P}$ can be written as :

$$
\mathbf{P}=(\mathbf{K R} \mathbf{K} \boldsymbol{t})
$$

$\mathbf{R}$ and $\boldsymbol{t}$ are the rotation and translation that link the camera frame to the 3-D Euclidean one. The most general form for the matrix of internal parameters $\mathbf{K}$ is :

$$
\mathbf{K}=\left(\begin{array}{ccc}
\alpha & r \alpha & u_{0} \\
0 & a \alpha & v_{0} \\
0 & 0 & 1
\end{array}\right)
$$

where $\alpha$ is the horizontal scale factor, $a$ is the ratio between the vertical and horizontal scale factors, $r$ is the image skew and $u_{0}$ and $v_{0}$ are the image coordinates of the principal point. 
When the aspect ratio $a$ is known and the image skew $r$ is zero (i.e. the image axes are orthogonal), the matrix of internal parameters depends only on 3 parameters and becomes:

$$
\mathbf{K}=\left(\begin{array}{ccc}
\alpha & 0 & u_{0} \\
0 & a \alpha & v_{0} \\
0 & 0 & 1
\end{array}\right)
$$

\section{$2.2 \quad$ Stratified calibration}

Autocalibration consists of recovering the metric information of the stereo rig. This information can be obtained through the recovery of the internal parameters and relative orientation and position of both cameras.

However, once the epipolar geometry of the stereo rig has been estimated and a projective basis has been defined, the metric information of the rig is fully encapsulated by the equation of the plane at infinity $\Pi_{\infty}$ and the equation of absolute conic $\boldsymbol{\Omega}_{\infty}[10,20]$.

\subsection{Notation}

In this paper we assume that the cameras of the stereo rig have constant parameters under the motion, and that the rig acquired a sequence of $n$ image pairs of a moving planar object.

We denote by $\Pi_{1}, \ldots, \Pi_{k}, \ldots, \Pi_{n}$ the geometric planes associated with the different positions of the planar object.

$\mathbf{H}_{i j}$ (resp. $\mathbf{H}_{i j}^{\prime}$ ) denote the homographies between the left (resp. right) image of the stereo rig in position $i$ and the left (resp. right) image of the stereo rig in position $j$. These $3 \times 3$ inter-image homographies can be computed from point correspondences.

We also denote by $\Gamma_{i j}$ the geometric Euclidean transformation that maps the points of $\Pi_{i}$ onto the points of $\Pi_{j}$. That is, if $M_{i}$ is a 3-D point of the object at position $i$ and $M_{j}$ the same point at position $j$, then these two points are related by $M_{j}=\Gamma_{i j}\left(M_{i}\right)$.

$\mathbf{A}^{\top}$ denotes the transpose of the matrix $\mathbf{A}$. [.] $\times$ denotes the matrix generating the cross product: $[\boldsymbol{x}]_{\times} \boldsymbol{y}=\boldsymbol{x} \wedge \boldsymbol{y}$.

\subsection{Organization of the paper}

The remainder of the paper is organized as follow. In Section 3, we explain how the epipolar geometry can easily be estimated from a sequence of image 
pairs of a planar object. In Section 4, the affine autocalibration is described and we show how the equation of the plane at infinity $\Pi_{\infty}$ can be estimated. The Euclidean autocalibration (estimation of the absolute conic $\boldsymbol{\Omega}_{\infty}$ ) is performed in Section 5. Section 6 shows some experiments with synthetic and real data in order to demonstrate the stability of the approach. Finally a brief discussion is given in Section 7.

\section{Projective calibration}

The projective calibration consists of estimating the epipolar geometry of the stereo rig. The epipolar geometry is assumed to be constant and can therefore be computed from many image pairs.

It is well known that the epipolar geometry cannot be estimated from a single image pair of a 3-D planar scene. However when the planar scene performs motions, all the image pairs (each corresponding to a different position of the planar scene) gathered by the stereo rig can be used and this makes the computation of the epipolar geometry possible.

The motions of the plane must be chosen so that they do not correspond to critical motions [13]. These are motions which are not sufficient to enable the epipolar geometry to be computed uniquely. In this case they are translations parallel to the plane of the scene, rotations orthogonal to the plane of the scene and combinations of the two. The plane is effectively fixed (as a set, not pointwise) relative to the rig under these motions.

The fundamental matrix $\mathbf{F}$ associated with the stereo rig is computed from all the left-to-right point correspondences from all the image pairs using a standard technique [19]. The projection matrices $\mathbf{P}$ and $\mathbf{P}^{\prime}$ associated with the left and right cameras respectively can then be derived [8]. Without loss of generality these two $3 \times 4$ matrices can be written as:

$$
\mathbf{P} \simeq\left(\begin{array}{ll}
\mathbf{I} & 0
\end{array}\right) \quad \mathbf{P}^{\prime} \simeq\left(\overline{\mathbf{P}}^{\prime} \boldsymbol{p}^{\prime}\right)
$$

where $\mathbf{I}$ is the $3 \times 3$ identity matrix, $\overline{\mathbf{P}}^{\prime}$ is a $3 \times 3$ matrix and $\boldsymbol{p}^{\prime}$ a 3 -vector.

Using point correspondences it is therefore possible to obtain a projective reconstruction of the points of the planes. It is also possible to estimate the projective coordinates $\boldsymbol{\pi}_{1}, \ldots, \boldsymbol{\pi}_{k}, \ldots, \boldsymbol{\pi}_{n}$ of the planes $\Pi_{1}, \ldots, \Pi_{k}, \ldots, \Pi_{n}$ associated with the different positions of the planar object. In the following, $\boldsymbol{\pi}_{k}$ is the 4-vector:

$$
\boldsymbol{\pi}_{k}=\left(\begin{array}{c}
\bar{\pi}_{k} \\
\alpha_{k}
\end{array}\right)
$$

where $\overline{\boldsymbol{\pi}}_{k}$ is a 3 -vector and $\alpha_{k}$ a real number. 


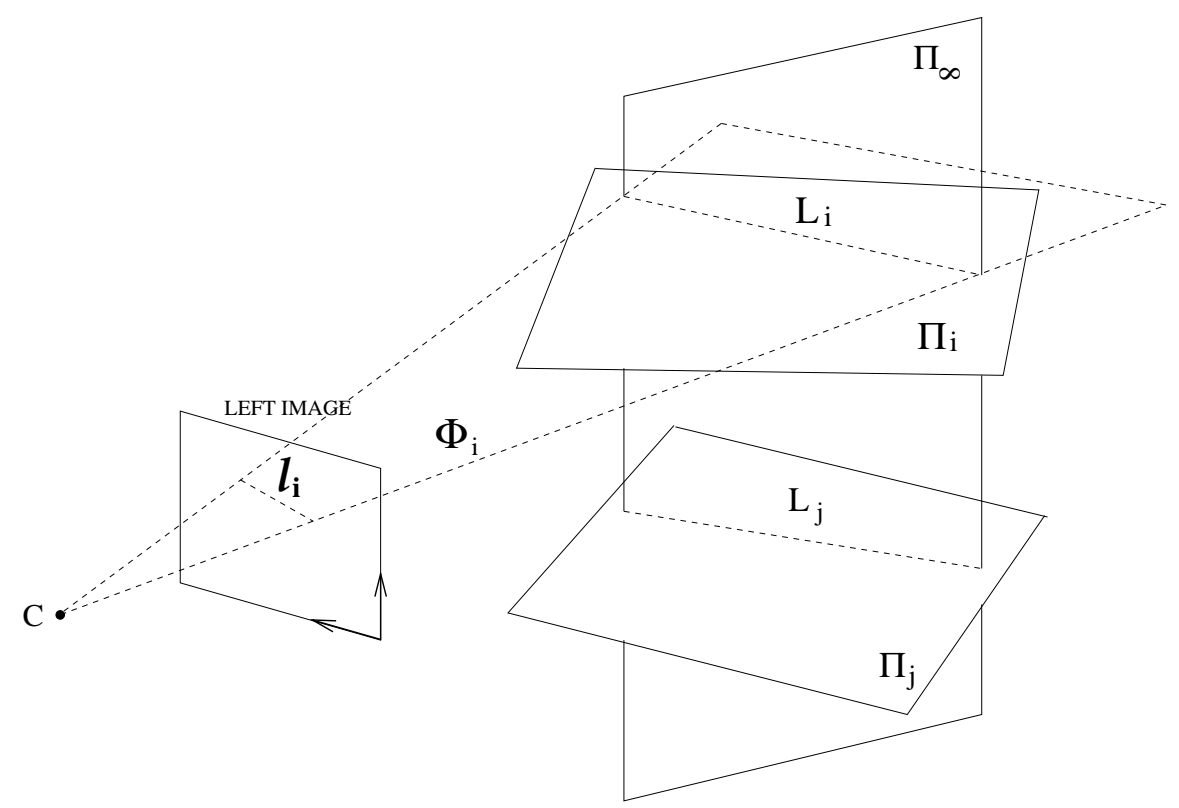

Fig. 1. The geometry of lines and planes involved in the affine autocalibration. The image line $\boldsymbol{l}_{i}$ is the vanishing line of the plane $\Pi_{i}$, which is the image of $L_{i}$.

\section{Affine calibration}

This section describes the affine autocalibration, which consists of estimating, in the projective basis determined previously (2), the coordinates $\boldsymbol{\pi}_{\infty}$ of the plane at infinity $\Pi_{\infty}$. For this purpose we use here the vanishing line of the observed plane in each left view, and show how quadratic constraints on the coordinates of this vanishing line can be derived.

We will use the fact that $\Pi_{\infty}$ is a particular plane: it is the only plane of projective space that remains globally invariant under any affine transformation, i.e. under the action of any affine transformation, any point lying on $\Pi_{\infty}$ has its image lying on $\Pi_{\infty}$ as well.

Let $L_{1}, \ldots, L_{k}, \ldots, L_{n}$ be the 3-D lines corresponding to the intersections of $\Pi_{\infty}$ with $\Pi_{1}, \ldots, \Pi_{k}, \ldots, \Pi_{n}$ respectively, (see Figure 1). We use the following result:

Proposition 1. Consider any two lines $L_{i}$ and $L_{j}$ among $L_{1}, \ldots, L_{n} . \Gamma_{i j}$ being the Euclidean transformation that maps $\Pi_{i}$ onto $\Pi_{j}$ as defined in Section 2.3, we have:

$$
L_{j}=\Gamma_{i j}\left(L_{i}\right)
$$


Proof: The intersection of two planes is preserved by a Euclidean transformation (or indeed a projective transformation). However, a Euclidean transformation has the additional property that $\Pi_{\infty}$ is fixed (as a set, not pointwise). Therefore, $L_{i}\left(\right.$ on $\left.\Pi_{\infty}\right)$ is mapped to $L_{j}\left(\right.$ on $\left.\Pi_{\infty}\right)$. In our notation this is written:

$$
\begin{aligned}
\Gamma_{i j}\left(L_{i}\right) & =\Gamma_{i j}\left(\Pi_{\infty} \cap \Pi_{i}\right) \\
& =\Gamma_{i j}\left(\Pi_{\infty}\right) \cap \Gamma_{i j}\left(\Pi_{i}\right) \\
& =\Pi_{\infty} \cap \Pi_{j} \\
& =L_{j}
\end{aligned}
$$

This proves that, for all $k, 1 \leq k \leq n, L_{k}$ is the same line of the planar object in the different positions of the object, namely the line at infinity on the scene plane. An important feature of the lines $L_{1}, \ldots, L_{k}, \ldots, L_{n}$ is that they are all contained in the plane $\Pi_{\infty}$ and therefore are coplanar. This provides a constraint that will be used to solve for $\boldsymbol{\pi}_{\infty}$. In fact we actually solve for the vanishing line $l_{k}$ of each plane $\Pi_{k}$ and parameterize the solution by $l_{1}$.

Let $\boldsymbol{l}_{k}$ be the vanishing line of $\Pi_{k}$ which is the image of $L_{k}$ in the left camera (see Figure 1). Let $\Phi_{k}$ be the 3-D plane going through $L_{k}$ and the optical centre $C$ of the left camera. The plane $\Phi_{k}$ also intersects the left image plane at $\boldsymbol{l}_{k}$ and it can easily be shown that, in the projective basis defined in Section 3, the

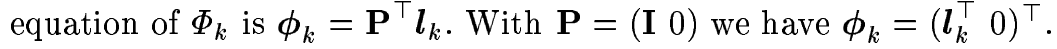

$L_{k}$ can be regarded as the intersection of $\Pi_{k}$ and $\Phi_{k} . \Pi_{k}$ and $\Phi_{k}$ define a pencil of planes that contains $L_{k}$, and $\Pi_{\infty}$ is in this pencil. $\Pi_{\infty}$ is therefore common to all pencils $\left(\Pi_{k}, \Phi_{k}\right)$. In other words, there exist some reals $\lambda_{1}, \lambda_{2}, \ldots, \lambda_{n}$ and $\mu_{1}, \mu_{2}, \ldots, \mu_{n}$ such that for all $k$ :

$$
\pi_{\infty}=\lambda_{k} \pi_{k}+\mu_{k} \phi_{k}
$$

Combining equation (4) for two pencils of planes $\left(\Pi_{i}, \Phi_{i}\right)$ and $\left(\Pi_{j}, \Phi_{j}\right)$ we obtain the constraint corresponding to the coplanarity on $\Pi_{\infty}$ of two lines $L_{i}$ and $L_{j}$ :

$$
\lambda_{i} \boldsymbol{\pi}_{i}+\mu_{i} \phi_{i}=\lambda_{j} \boldsymbol{\pi}_{j}+\mu_{j} \phi_{j}
$$

Equation (5) means that $\boldsymbol{\pi}_{i}, \phi_{i}, \boldsymbol{\pi}_{j}$ and $\boldsymbol{\phi}_{j}$ are linearly dependent and therefore is equivalent to $\operatorname{det}\left(\boldsymbol{\pi}_{i}, \boldsymbol{\pi}_{j}, \boldsymbol{\phi}_{i}, \boldsymbol{\phi}_{j}\right)=0$. Using (3) for $\boldsymbol{\pi}_{k}$, the condition for two lines $L_{i}$ and $L_{j}$ being coplanar becomes:

$$
\left|\begin{array}{cccc}
\overline{\boldsymbol{\pi}}_{i} & \overline{\boldsymbol{\pi}}_{j} & \boldsymbol{l}_{i} & \boldsymbol{l}_{j} \\
\alpha_{i} & \alpha_{j} & 0 & 0
\end{array}\right|=0
$$

The lines $\boldsymbol{l}_{1}, \ldots, \boldsymbol{l}_{k}, \ldots, \boldsymbol{l}_{n}$ represent the corresponding vanishing lines of the plane in the different images. Since all $l_{k}$ are images of $L_{1}$ on $\Pi_{1}$, we have:

$$
l_{k}=\mathbf{H}_{1 k}^{-\top} \boldsymbol{l}_{1}
$$


We can therefore express all the lines $\boldsymbol{l}_{2}, \ldots, \boldsymbol{l}_{n}$ with respect to $\boldsymbol{l}_{1}$. Expanding the determinant (6), we obtain the following quadratic equation:

$$
l_{1}^{\top} \mathbf{C}_{i j}^{\star} l_{1}=0
$$

where $\mathbf{C}_{i j}^{\star}$ is a $3 \times 3$ symmetric matrix such that $\mathbf{C}_{i j}^{\star}=\frac{\mathbf{A}_{i j}+\mathbf{A}_{i j}^{\top}}{2}$ and $\mathbf{A}_{i j}$ is a $3 \times 3$ matrix defined by $\mathbf{A}_{i j}=\mathbf{H}_{1 j}^{-1}\left[\alpha_{j} \overline{\boldsymbol{\pi}}_{i}-\alpha_{i} \overline{\boldsymbol{\pi}}_{j}\right]_{\times} \mathbf{H}_{1 i}^{-\top}$.

The coplanarity of $L_{i}$ and $L_{j}$ therefore defines a quadratic constraint on $\boldsymbol{l}_{1}$. Once $\boldsymbol{l}_{1}$ is estimated, the lines $\boldsymbol{l}_{2}, \ldots, \boldsymbol{l}_{n}$ are estimated from (7), and the equations of the planes $\phi_{1}, \ldots, \phi_{n}$ as well.

We will see that only the lines $l_{1}, \ldots, l_{n}$ are required for Euclidean autocalibration. However $\boldsymbol{\pi}_{\infty}$ can also be estimated. $\boldsymbol{\pi}_{\infty}$ is computed as the common plane to all pencils of planes $\left(\Pi_{k}, \Phi_{k}\right)$. In practice, $\pi_{\infty}$ is computed by solving the linear system defined by equations (4) where the unknowns are $\boldsymbol{\pi}_{\infty}$ and the reals $\lambda_{1}, \ldots, \lambda_{n}$ and $\mu_{1}, \ldots, \mu_{n}$. For $n$ positions, this linear system has $2 n+4$ unknowns ( $n \lambda$ 's, $n \mu$ 's and 4 for $\pi_{\infty}$ ) and $4 n$ equations, and these can be solved using an SVD approach. To conclude:

- with 2 views of the planar object, we obtain a single constraint $\mathbf{C}_{12}^{\star}$ and there is a one-parameter family of solutions for $l_{1}$ (all the lines of the conic $\mathbf{C}_{12}^{\star}$ ). Therefore there is a one-parameter family of solutions for $\boldsymbol{\pi}_{\infty}$;

- with 3 views of the planar object, we obtain 3 independent constraints $\mathbf{C}_{12}^{\star}$, $\mathbf{C}_{13}^{\star}$ and $\mathbf{C}_{23}^{\star}$, and $\boldsymbol{l}_{1}$ corresponds to the common intersection of these conics. The solution of the equations (8) can be found in Annex A. $\boldsymbol{\pi}_{\infty}$ is thus determined uniquely.

\section{Euclidean calibration}

Let $\Omega_{\infty}$ be the absolute conic and $\omega_{\infty}$ and $\omega_{\infty}^{\prime}$ its projection onto the left and right camera respectively. A fundamental property of $\boldsymbol{\Omega}_{\infty}, \omega_{\infty}$ and $\omega_{\infty}^{\prime}$ is that they are all invariant to Euclidean transformations (provided that the internal parameters of the cameras are constant). Euclidean autocalibration consists of estimating the coordinates of $\boldsymbol{\Omega}_{\infty}$. It is also equivalent, given $\Pi_{\infty}$, to estimating the equation of one of the projections of $\boldsymbol{\Omega}_{\infty}$. We can choose, for instance, to estimate its left projection $\boldsymbol{\omega}_{\infty}$ whose expression is $\boldsymbol{\omega}_{\infty}=\left(\mathbf{K K}^{\top}\right)^{-1}$ where $\mathbf{K}$ is the matrix of internal parameters of the left camera.

Consider the (complex) circular points $\mathcal{I}_{k}$ and $\overline{\mathcal{I}}_{k}$ of the plane $\Pi_{k}$. By definition $\mathcal{I}_{k}$ and $\overline{\mathcal{I}}_{k}$ are the intersections of $\Pi_{k}$ with $\boldsymbol{\Omega}_{\infty}$ and therefore are also the intersections of $L_{k}$ with $\boldsymbol{\Omega}_{\infty}$. Let $\boldsymbol{I}_{k}$ and $\overline{\boldsymbol{I}}_{k}$ be the projections of $\mathcal{I}_{k}$ and $\overline{\mathcal{I}}_{k}$ onto the left camera. As a consequence, $\boldsymbol{I}_{k}$ and $\overline{\boldsymbol{I}}_{k}$ are the intersections of $\boldsymbol{l}_{k}$ and $\boldsymbol{\omega}_{\infty}$. Solving for $\boldsymbol{\omega}_{\infty}$ then consists of the following steps: 

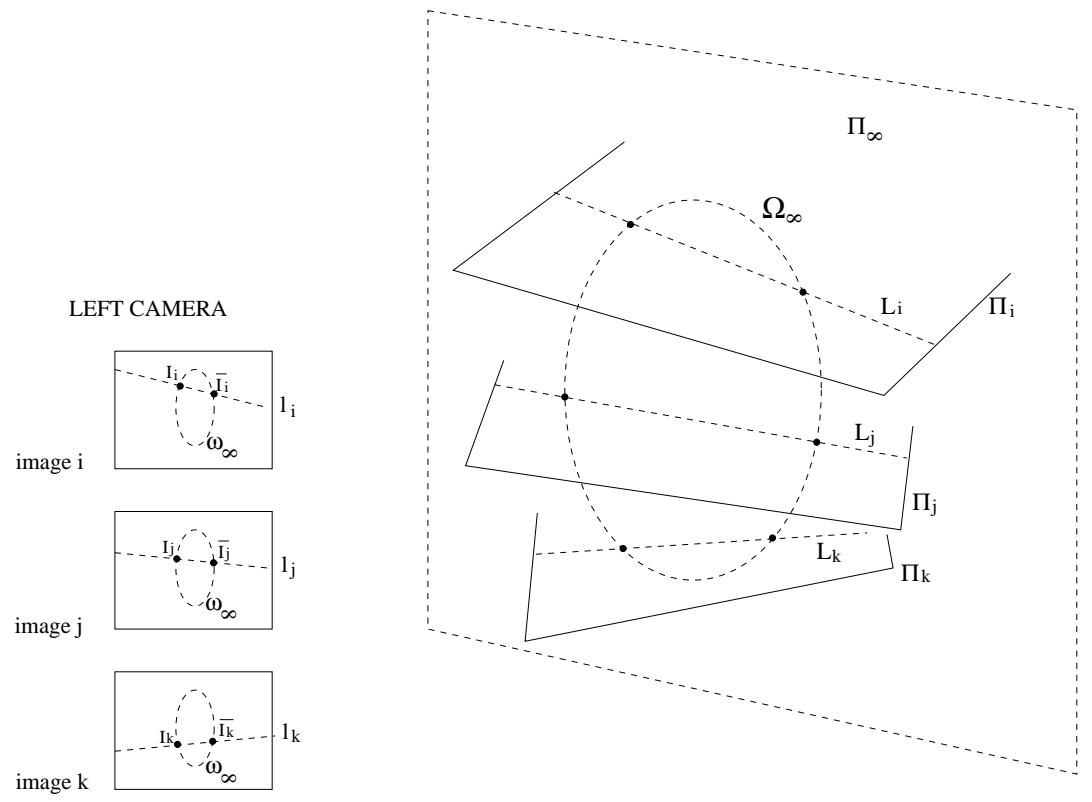

Fig. 2. The circular points lie on the absolute conic $\boldsymbol{\Omega}_{\infty}$

1. Use the constraint that the points $\boldsymbol{I}_{k}$ and $\overline{\boldsymbol{I}}_{k}$ lie on the lines $\boldsymbol{l}_{k}$ estimated by the affine autocalibration;

2. Express the constraint that all $\boldsymbol{I}_{k}$ and $\overline{\boldsymbol{I}}_{k}$ lie on the same conic $\boldsymbol{\omega}_{\infty}$;

3. Estimate $\boldsymbol{\omega}_{\infty}$ from all $\boldsymbol{I}_{k}$ and $\overline{\boldsymbol{I}}_{k}$;

4. Compute $\mathbf{K}$ from $\boldsymbol{\omega}_{\infty}$.

Let $\boldsymbol{p}_{1}$ and $\boldsymbol{q}_{1}$ be two real points lying on $\boldsymbol{l}_{1} . \boldsymbol{I}_{1}$ can be parameterized by a complex $\lambda$ such that $\boldsymbol{I}_{1}=\boldsymbol{q}_{1}+\lambda \boldsymbol{p}_{1}$. As all $\boldsymbol{I}_{k}$ and $\overline{\boldsymbol{I}}_{k}$ belong to the planar object, they are related by the inter-image homographies $\mathbf{H}_{i j}$ and therefore we have for all $k$ :

$$
\begin{aligned}
\boldsymbol{I}_{k} & =\mathbf{H}_{1 k} \boldsymbol{I}_{1}=\mathbf{H}_{1 k} \boldsymbol{q}_{1}+\lambda \mathbf{H}_{1 k} \boldsymbol{p}_{1} \\
\overline{\boldsymbol{I}}_{k} & =\mathbf{H}_{1 k} \overline{\boldsymbol{I}}_{1}=\mathbf{H}_{1 k} \boldsymbol{q}_{1}+\bar{\lambda} \mathbf{H}_{1 k} \boldsymbol{p}_{1}
\end{aligned}
$$

A constraint can be expressed on $\lambda$ that all points $\boldsymbol{I}_{k}$ and $\overline{\boldsymbol{I}}_{k}$ lie on the same conic $\boldsymbol{\omega}_{\infty}$. We will consider first the case of unrestricted $\mathbf{K}$.

\subsection{General calibration $\mathbf{K}$}

Consider any 3 positions of the planar object associated with the planes $\Pi_{i}, \Pi_{j}$ and $\Pi_{k}$ and the projections $\boldsymbol{I}_{i}, \overline{\boldsymbol{I}}_{i}, \boldsymbol{I}_{j}, \overline{\boldsymbol{I}}_{j}, \boldsymbol{I}_{k}$ and $\overline{\boldsymbol{I}}_{k}$ of their circular points onto the left camera. 
Let $\boldsymbol{Y}_{i j}, \boldsymbol{Y}_{i k}$ and $\boldsymbol{Y}_{j k}$ be the respective intersections of the lines $\left(\boldsymbol{I}_{i} \overline{\boldsymbol{I}}_{j}\right)$ and $\left(\overline{\boldsymbol{I}}_{i} \boldsymbol{I}_{j}\right),\left(\boldsymbol{I}_{i} \overline{\boldsymbol{I}}_{k}\right)$ and $\left(\overline{\boldsymbol{I}}_{i} \boldsymbol{I}_{k}\right),\left(\boldsymbol{I}_{j} \overline{\boldsymbol{I}}_{k}\right)$ and $\left(\overline{\boldsymbol{I}}_{j} \boldsymbol{I}_{k}\right)$. One can show that the expression of $\boldsymbol{Y}_{i j}$ is:

$$
\begin{aligned}
\boldsymbol{Y}_{i j} & \simeq\left(\boldsymbol{I}_{i} \wedge \overline{\boldsymbol{I}}_{j}\right) \wedge\left(\boldsymbol{I}_{j} \wedge \overline{\boldsymbol{I}}_{i}\right) \\
& \simeq A_{i j} u+B_{i j} v+C_{i j}
\end{aligned}
$$

where $A_{i j}, B_{i j}$ and $C_{i j}$ are three reals depending only on the entries of $\boldsymbol{p}_{1}, \boldsymbol{q}_{1}$, $\mathbf{H}_{1 i}$ and $\mathbf{H}_{1 j}$, and $u$ and $v$ are two real numbers such that $u=\lambda \bar{\lambda}$ and $v=\lambda+\bar{\lambda}$.

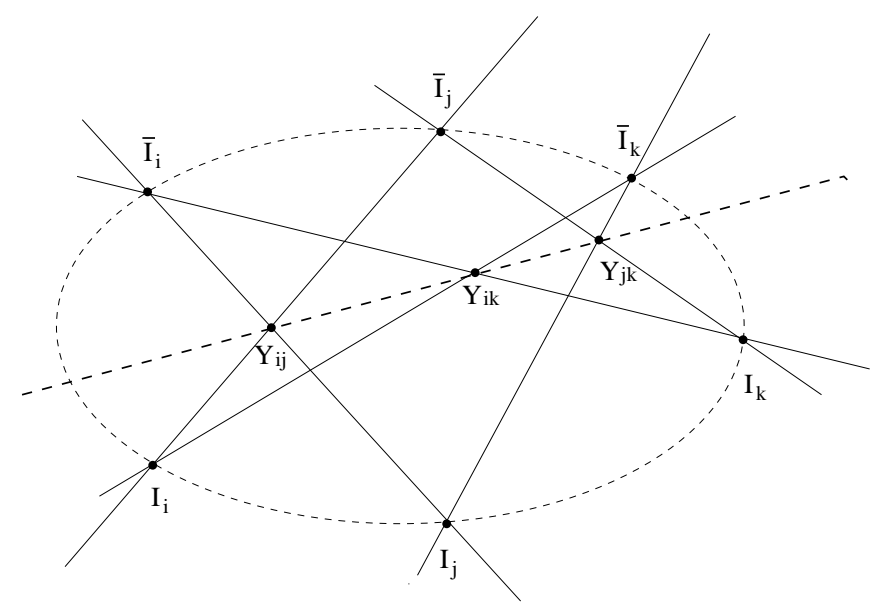

Fig. 3. Pascal's theorem : condition for 6 points to lie on a conic.

From Pascal's theorem, the six points $\boldsymbol{I}_{i}, \overline{\boldsymbol{I}}_{i}, \boldsymbol{I}_{j}, \overline{\boldsymbol{I}}_{j}, \boldsymbol{I}_{k}$ and $\overline{\boldsymbol{I}}_{k}$ lie on the same conic if and only if $\boldsymbol{Y}_{i k}, \boldsymbol{Y}_{j k}$ and $\boldsymbol{Y}_{i j}$ lie on a line (see Figure 3). This can be expressed as:

$$
\operatorname{det}\left(\boldsymbol{Y}_{i k}, \boldsymbol{Y}_{j k}, \boldsymbol{Y}_{i j}\right)=0
$$

Using the expression obtained in (10) for $\boldsymbol{Y}_{i j}$ it is clear that (11) - and therefore the constraint that the points $\boldsymbol{I}_{i}, \overline{\boldsymbol{I}}_{i}, \boldsymbol{I}_{j}, \overline{\boldsymbol{I}}_{j}, \boldsymbol{I}_{k}$ and $\overline{\boldsymbol{I}}_{k}$ are on a conic - is a cubic equation in $u$ and $v$ :

$$
\Gamma_{i j k}(u, v)=\sum_{N=0}^{N \leq 3} \sum_{m=0}^{m \leq N} \gamma_{m, N-m} u^{m} v^{N-m}
$$

where $\gamma_{m, N-m}$ are some real numbers depending only on the entries of $\boldsymbol{p}_{1}, \boldsymbol{q}_{1}$, $\mathbf{H}_{1 i}, \mathbf{H}_{1 j}$ and $\mathbf{H}_{1 k}$.

From 4 views, it is therefore possible to obtain 4 cubic constraints $\Gamma_{i j k}(u, v)$ such as (12). Solving simultaneously these cubic constraints [15] gives a solution for $(u, v)$ from which $\lambda$ and hence $\omega_{\infty}$ may be computed. 


\subsection{Zero skew, known aspect ratio}

In the case of a 3-parameter projective camera as described by the model (1), skew is zero and the aspect ratio $a$ is known. These constraints can be imposed by introducing two complex points $\boldsymbol{J}$ and $\overline{\boldsymbol{J}}$ such that $\boldsymbol{J}=\left(\begin{array}{lll}1 & a i & 0\end{array}\right)^{\top}$ and $\overline{\boldsymbol{J}}=(1-a i 0)^{\top}$. Then if skew is zero and the aspect ratio $a$ is known $\boldsymbol{J}$ and $\overline{\boldsymbol{J}}$ lie on $\boldsymbol{\omega}_{\infty}$ (the intersection of $\boldsymbol{\omega}_{\infty}$ with the line at infinity in the image).

The same approach as in the general case described above can be used. Using any two positions $i$ and $j$ of the planar object, a constraint derived from Pascal's theorem can be expressed that the 6 points $\boldsymbol{I}_{i}, \overline{\boldsymbol{I}}_{i}, \boldsymbol{I}_{j}, \overline{\boldsymbol{I}}_{j}, \boldsymbol{J}$ and $\overline{\boldsymbol{J}}$ lie on the same conic $\boldsymbol{\omega}_{\infty}$. Including $\boldsymbol{J}$ and $\overline{\boldsymbol{J}}$ reduces the number of views required to solve for $\boldsymbol{\omega}_{\infty}$. In this case the constraint (11) has the form:

$$
(\lambda-\bar{\lambda})^{2} \boldsymbol{x}^{\top} \mathbf{Q}_{i j} \boldsymbol{x}=0
$$

where $\boldsymbol{x}$ is a real 3 -vector such that $\boldsymbol{x}=(\lambda \bar{\lambda}, \lambda+\bar{\lambda}, 1)$ and $\mathbf{Q}_{i j}$ is a $3 \times 3$ symmetric matrix that depends only on the entries of $\boldsymbol{p}_{1}, \boldsymbol{q}_{1}, \mathbf{H}_{1 i}, \mathbf{H}_{1 j}$ and the aspect ratio a. As $\lambda$ is a non-real complex number, then $\lambda \neq \bar{\lambda}$ and the constraint reduces to:

$$
\boldsymbol{x}^{\top} \mathbf{Q}_{i j} \boldsymbol{x}=0
$$

Then from two views we obtain a quadratic constraint on $\boldsymbol{x}$. From 3 views or more, we obtain therefore at least 3 independent conics $\mathbf{Q}_{i j}$ corresponding to the quadratic constraints (13). The intersection of these conics gives, when the motions of the planar object are general, a unique solution for $\boldsymbol{x}$.

Once $\boldsymbol{x}$ is computed (see details in Annex A), $\lambda$ is known and then all the points $\boldsymbol{I}_{k}, \overline{\boldsymbol{I}}_{k}$ can be estimated as well. $\boldsymbol{\omega}_{\infty}$ can then be computed as the conic going through all the points $\boldsymbol{I}_{k}, \overline{\boldsymbol{I}}_{k}$ and $\boldsymbol{J}$ and $\overline{\boldsymbol{J}}$.

Finally $\mathbf{K}$ is estimated by the Cholesky decomposition of $\boldsymbol{\omega}_{\infty}=\left(\mathbf{K K}^{\top}\right)^{-1}$.

\subsection{Summary of the autocalibration algorithm}

The complete algorithm can be summarized as follow:

1. Compute the fundamental matrix $\mathbf{F}$ and the projective coordinates $\boldsymbol{\pi}_{1}, \ldots, \boldsymbol{\pi}_{n}$ of the planes $\Pi_{1}, \ldots, \Pi_{n}$;

2. Estimate the inter-image homographies $\mathbf{H}_{i j}$;

3. Affine autocalibration: solve the quadratic constraints (8) for $l_{1}$;

4. Euclidean autocalibration: solve the quadratic constraints (13). Compute $\lambda$, $\boldsymbol{I}_{k}$ and $\overline{\boldsymbol{I}}_{\boldsymbol{k}}$ with (9). Then compute $\boldsymbol{\omega}_{\infty}$ as the conic going through all $\boldsymbol{I}_{\boldsymbol{k}}$ and $\overline{\boldsymbol{I}}_{k}$ and finally compute $\mathbf{K}$ by Cholesky decomposition;

5. Bundle adjustment (optional): minimization of point backprojection errors onto the left and right cameras of the 3-D planar scene at its different locations. 


\section{$6 \quad$ Experiments}

The stereo autocalibration algorithm has been implemented in matlab and applied to both synthetic and real data.

\subsection{Synthetic data}
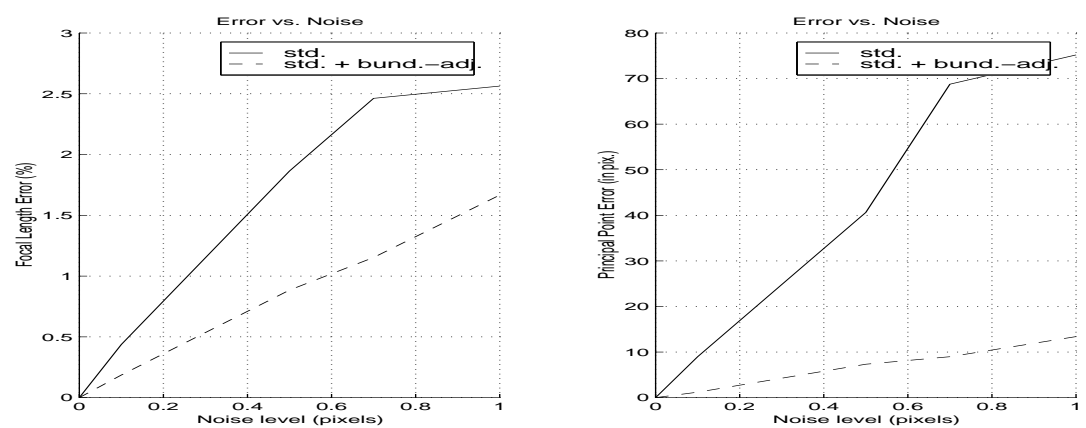

Fig. 4. Errors in the estimation of focal length (in \%) and of principal point (in pix.) vs. level of noise.
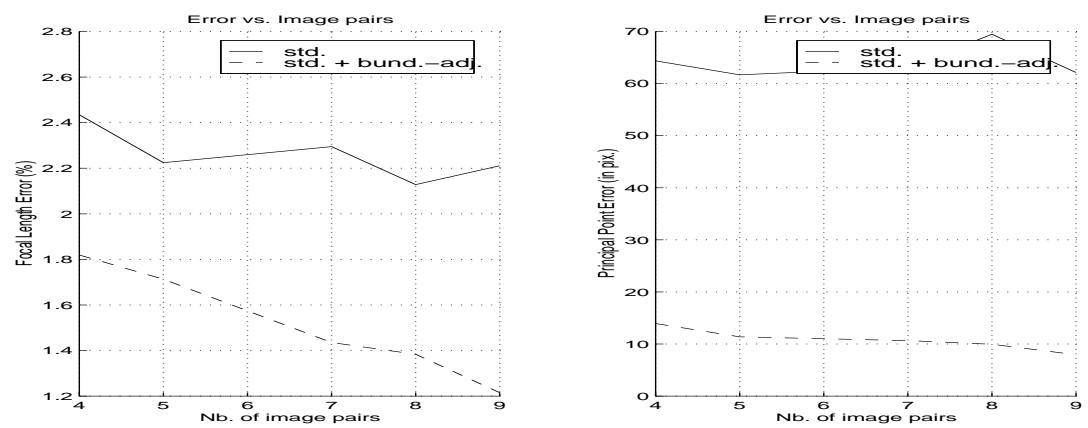

Fig. 5. Errors in the estimation of focal length (in \%) and of principal point (in pix.) vs. number of image pairs.

Experiments with simulated data are carried out in order to assess the stability of the method against measurement noise.

A synthetic 3-D planar scene consisting of 100 points is generated and placed at different locations in 3-D space. The 3-D points of each position are projected 
onto the cameras of a stereo rig and Gaussian noise with varying standard deviation $\sigma$ (from 0.0 to 1.0 pixel) is added to the image point locations. The cameras have a nominal focal length $f$ of 1200 pixels, unit aspect ratio and zero image skew and the image size is $512 \times 512$. Image point locations are normalized as described in [6] and inter-image homographies $\mathbf{H}_{i j}$ are estimated. The autocalibration is then computed 100 times for each $\sigma$.

Figure 4 shows the resulting accuracy with varying noise and 7 image pairs. Figure 5 shows the resulting accuracy with a fixed noise level of 0.7 pix. and a varying number of image pairs.

The experiments show that the estimation provided by the method is quite accurate. Even for a level of noise of 1.0 pix., the error in the estimation of the focal length is less than $2.5 \%$. Moreover the approach gives sufficiently stable and accurate results to initialize a bundle adjustment procedure. With such a procedure, the accuracy of the estimation of both the focal length and the location of the principal point is increased as shown in Figures 4 and 5.

\subsection{Real data}
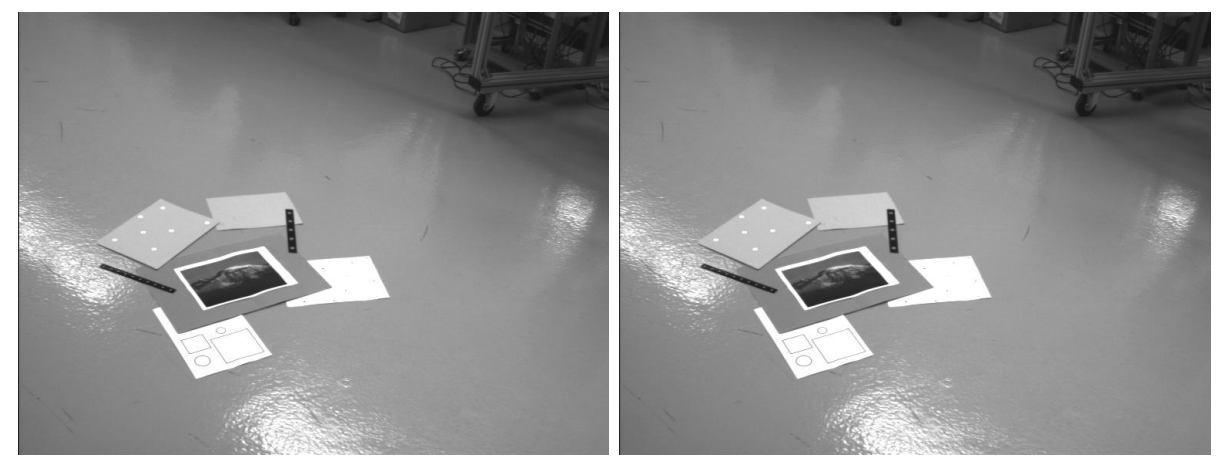

Fig. 6. One of the seven pairs gathered by the stereo rig

We gathered 7 image pairs of a planar scene (see Figure 6 ) with a stereo rig. Thirty points are matched between all images and the autocalibration algorithm applied using 4 to 7 image pairs from the whole sequence. In order to show the efficiency of the method, we show results before and after applying the bundle-adjustment procedure. The results are shown in Figure 7 where they are compared with the results of an off-line calibration [3].

As the number of views increases the estimated values approach ground truth. Although we used few points and all matches were made by hand, the method gives acceptable results. The bundle-adjustment procedure, initialized with these results, provides accurate enough calibration for metric reconstruction purposes. 


\begin{tabular}{|c|c|c|c|c|c|c|c|}
\hline \multirow{2}{*}{$\begin{array}{l}\text { Autocalibration } \\
n b . \text { of image pairs }\end{array}$} & \multirow[b]{2}{*}{ method } & \multicolumn{3}{|c|}{ left camera } & \multicolumn{3}{|c|}{ right camera } \\
\hline & & $f$ & $u_{0}$ & $v_{0}$ & $f$ & $u_{0}$ & $v_{0}$ \\
\hline \multirow[t]{2}{*}{4 image pairs } & std. & 1008 & 420 & 275 & 1076 & 298 & 313 \\
\hline & ind. - adj. & 1058 & 373 & 304 & 1065 & 264 & 276 \\
\hline \multirow[t]{2}{*}{5 image pairs } & std. & 1088 & 503 & 286 & 1060 & 399 & 290 \\
\hline & $w /$ bund. $-a d j$. & 1060 & 421 & 221 & 1025 & 306 & 254 \\
\hline \multirow[t]{2}{*}{6 image pairs } & std. & 1008 & 320 & 300 & 1090 & 278 & 343 \\
\hline & $w /$ bund.-adj. & 1020 & 399 & 274 & 1036 & 291 & 294 \\
\hline \multirow[t]{2}{*}{7 image pairs } & std. & 1048 & 345 & 247 & 1116 & 212 & 254 \\
\hline & $w /$ bund.-adj. & 1022 & 400 & 279 & 1041 & 301 & 290 \\
\hline Off-line calibration & & 1030 & 399 & 269 & 1045 & 30 & 28 \\
\hline
\end{tabular}

Fig. 7. Results of autocalibration algorithm for the real data of Figure 6 using different numbers of image pairs and off-line calibration.

\section{Conclusion}

We describe in this paper a new method for autocalibrating a stereo rig from several views of a plane.

We show that the epipolar geometry of the rig can easily be estimated with a planar scene in motion. We use the constraint that the projections of the circular points of a 3-D plane must lie on the image of the absolute conic. Then the autocalibration is performed by applying a stratified approach. Both autocalibration steps -affine and Euclidean-involve a set of quadratic constraints and we therefore designed a conic intersection method to solve for them.

Futhermore, our approach provides an algebraic solution (i.e. non-iterative) to Trigg's planar method [17] when vanishing lines are known, and this could be used for autocalibrating a camera from a monocular sequence of planes.

\section{A Intersection of conics}

Let $\mathbf{C}_{1}, \ldots, \mathbf{C}_{k}, \ldots, \mathbf{C}_{n}$ be $n$ conics $(n \geq 3)$ represented as $3 \times 3$ matrices. Let us suppose that these conics have a common intersection $\boldsymbol{x}$. For each $k$ we have:

$$
\boldsymbol{x}^{\top} \mathbf{C}_{k} \boldsymbol{x}=0
$$

Consider any two conics $\mathbf{C}_{i}$ and $\mathbf{C}_{j}$. Let $\nu_{0}$ be a real number such that $\operatorname{det}\left(\mathbf{C}_{i}+\nu_{0} \mathbf{C}_{j}\right)=0\left(\nu_{0}\right.$ always exists because $\nu \mapsto \operatorname{det}\left(\mathbf{C}_{i}+\nu \mathbf{C}_{j}\right)$ is a degreethree polynomial with real factors). Let $\mathbf{D}_{i j}$ be such that $\mathbf{D}_{i j}=\mathbf{C}_{i}+\nu_{0} \mathbf{C}_{j}$. Then $\mathbf{D}_{i j}$ belongs to the pencil of conics generated by $\mathbf{C}_{i}$ and $\mathbf{C}_{j}$ and is degenerate $\left(\operatorname{det}\left(\mathbf{D}_{i j}\right)=0\right)$. Moreover $\boldsymbol{x}$ belongs to $\mathbf{D}_{i j}$ because:

$$
\begin{aligned}
\boldsymbol{x}^{\top} \mathbf{D}_{i j} \boldsymbol{x} & =\boldsymbol{x}^{\top}\left(\mathbf{C}_{i}+\nu_{0} \mathbf{C}_{j}\right) \boldsymbol{x} \\
& =\boldsymbol{x}^{\top} \mathbf{C}_{i} \boldsymbol{x}+\nu_{0} \boldsymbol{x}^{\top} \mathbf{C}_{j} \boldsymbol{x}=0
\end{aligned}
$$




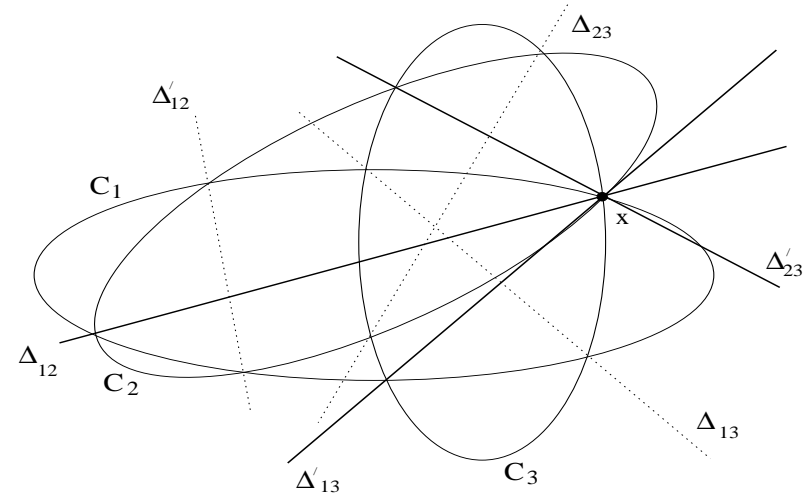

Fig. 8. Intersection of conics

As a degenerate conic, $\mathbf{D}_{i j}$ is the union of two lines $\Delta_{i j}$ and $\Delta_{i j}^{\prime}$ and therefore $\boldsymbol{x}$ lies on one (at least) of these two lines. As a consequence, $\boldsymbol{x}$ can be estimated as the common intersection of all the pairs of lines $\Delta_{i j}$ and $\Delta_{i j}^{\prime}$.

Therefore the method we propose for solving simultaneously the quadratic constraints defined by the matrices $\mathbf{C}_{k}$ consists of the following steps:

- compute the degenerate conics $\mathbf{D}_{i j}$ and their associated pairs of lines $\Delta_{i j}$ and $\Delta_{i j}^{\prime}$. In practice, it is not necessary to compute all the possible $\mathbf{D}_{i j}$, we can choose to compute only $n$ of them;

- intersect the pairs of lines $\Delta_{i j}$ and $\Delta_{i j}^{\prime}$, that is, find a point $\boldsymbol{x}$ such that it belongs to one line at least of each pair of lines $\Delta_{i j}$ and $\Delta_{i j}^{\prime}$. It is worth noticing that when data are noisy, the lines do not exactly intersect at the same point and an approach similar to linear least squares can be used to find the closest point $\boldsymbol{x}$ to all pairs of lines.

\section{Acknowledgements}

We are grateful for discussions with Frederik Schaffalitzky.

\section{References}

1. M. Armstrong, A. Zisserman, and R. Hartley. Self-calibration from image triplets. In B. Buxton and R. Cipolla, editors, Proceedings of the 4 th European Conference on Computer Vision, Cambridge, England, volume 1064 of Lecture Notes in Computer Science, pages 3-16. Springer-Verlag, April 1996. 
2. F. Devernay and O. Faugeras. From projective to euclidean reconstruction. In Proceedings of the Conference on Computer Vision and Pattern Recognition, San Francisco, California, USA, pages 264-269, June 1996.

3. O. Faugeras. Three-Dimensional Computer Vision - A Geometric Viewpoint. Artificial intelligence. The MIT Press, Cambridge, MA, USA, Cambridge, MA, 1993.

4. O. Faugeras. Stratification of three-dimensional vision: Projective, affine and metric representations. Journal of the Optical Society of America, 12:465-484, 1995.

5. O.D. Faugeras, Q.T. Luong, and S.J. Maybank. Camera self-calibration: Theory and experiments. In G. Sandini, editor, Proceedings of the 2nd European Conference on Computer Vision, Santa Margherita Ligure, Italy, pages 321-334. Springer-Verlag, May 1992.

6. R. Hartley. In defence of the 8-point algorithm. In Proceedings of the 5th International Conference on Computer Vision, Cambridge, Massachusetts, USA, pages 1064-1070, June 1995.

7. R.I. Hartley. Euclidean reconstruction from uncalibrated views. In Proceeding of the DARPA-ESPRIT workshop on Applications of Invariants in Computer Vision, Azores, Portugal, pages 187-202, October 1993.

8. R.I. Hartley. Projective reconstruction and invariants from multiple images. IEEE Transactions on Pattern Analysis and Machine Intelligence, 16(10):1036-1041, October 1994.

9. A. Heyden and K. Åström. Euclidean reconstruction from constant intrinsic parameters. In Proceedings of the 13th International Conference on Pattern Recognition, Vienna, Austria, volume I, pages 339-343, August 1996.

10. R. Horaud and G. Csurka. Self-calibration and euclidean reconstruction using motions of a stereo rig. In Proceedings of the 6th International Conference on Computer Vision, Bombay, India, pages 96-103, January 1998.

11. D. Liebowitz, A. Criminisi, and A. Zisserman. Creating architectural models from images. In Proc. EuroGraphics, volume 18, pages 39-50, September 1999.

12. Q.T. Luong and T. Vieville. Canonic representations for the geometries of multiple projective views. Technical report, University of California, Berkeley, EECS, Cory Hall 211-215, University of California, Berkeley, CA 94720, October 1993.

13. S. Maybank. Theory of Reconstruction from Image Motion. Springer-Verlag, 1993.

14. M. Pollefeys and L. Van Gool. A stratified approach to metric self-calibration. In Proceedings of the Conference on Computer Vision and Pattern Recognition, Puerto Rico, USA, pages 407-412. IEEE Computer Society Press, June 1997.

15. J.G. Semple and G.T. Kneebone. Algebraic Projective Geometry. Oxford Science Publication, 1952.

16. P. Sturm and S. Maybank. On plane-based camera calibration: A general algorithm, singularities, applications. Proceedings of the Conference on Computer Vision and Pattern Recognition, Fort Collins, Colorado, USA, 1999.

17. B. Triggs. Autocalibration from planar scenes. In Proceedings of the 5th European Conference on Computer Vision, Freiburg, Germany, 1998.

18. Z. Zhang. A flexible new technique for camera calibration. In Proceedings of the 7th International Conference on Computer Vision, Kerkyra, Greece, September 1999.

19. Z. Zhang, R. Deriche, O. D. Faugeras, and Q-T. Luong. A robust technique for matching two uncalibrated images through the recovery of the unknown epipolar geometry. Artificial Intelligence, 78(1-2):87-119, October 1995.

20. A. Zisserman, P.A. Beardsley, and I.D. Reid. Metric calibration of a stereo rig. In Workshop on Representation of Visual Scenes, Cambridge, Massachusetts, USA, pages $93-100$, June 1995. 